\section{Measuring surface vibrations of musical instruments using an inexpensive digital holography device}

\author{
Nazif Demoli, MEMBER SPIE \\ Ivan Demoli \\ Institute of Physics \\ Bijenička c. 46 \\ HR-10000 Zagreb, Croatia \\ E-mail: demoli@ifs.hr
}

\begin{abstract}
A new device for measuring surface vibrations of musical instruments is presented. The architecture of the device is based on a quasi-Fourier digital holography setup with inexpensive commercial elements capable of recording large digital holograms. Experimental results showing vibration modes for several musical instruments are given. The results demonstrate fringe pattern quality as well as the testing possibilities in tuning the musical instruments. (๑) 2005 Society of Photo-Optical Instrumentation Engineers.
\end{abstract}

[DOI: 10.1117/1.2042467]

Subject terms: digital holography; time-averaged holographic interferometry; vibrating membrane.

Paper 050232 L received Apr. 4, 2005; accepted for publication Aug. 1, 2005; appeared online Aug. 2, 2005; published online Sep. 13, 2005.

The quality of the radiated sound of a musical instrument depends mostly on the structural vibrations of its resonator. Since the structural vibrations manifest themselves primarily through the surface vibrations, these have been studied applying numerical modeling and diverse experimental techniques. ${ }^{1}$ A known numerical method for calculating vibrational modes is finite element analysis. ${ }^{2}$ The experimental approaches include pointwise methods such as those using accelerometers ${ }^{3}$ and full-field methods such as time-averaged holographic interferometry ${ }^{4}$ and electronic speckle pattern interferometry. ${ }^{5}$ However, these experimental techniques are time and means consuming since they use on one hand complicated procedures and on the other hand expensive equipment such as special monochrome CCD cameras or high-quality optical elements. We propose a new device for surface vibration measurements that is simple, fast, and inexpensive, yet provides full-field and non-contact measurements. Our approach is based on using a quasi-Fourier digital holography setup with commercial inexpensive elements such as a color digital camera and large plane mirrors.

In digital holography with a quasi-Fourier setup, ${ }^{6,7}$ both a reference point source and an object are placed at the input plane and an array photodetector at the hologram plane. Thus, digital holograms are recorded optically and stored in computer memory as image files of primary interference fringes. Two symmetrical hologram reconstructions are obtained numerically by calculating the Fourier trans-

0091-3286/2005/\$22.00 @ 2005 SPIE form of the recorded data. If we denote the dynamic object wave front with $s(x, y, t)$ and the position of the point source with $(X, Y)$, then the input can be described as: $U(x, y, t)=\delta(x-X, y-Y)+s(x, y, t)$. If only out-of-plane harmonic vibrations of the object surface occur and the illumination is normal to the surface, then $s(x, y, t)$ $=s(x, y) \exp [i(4 \pi / \lambda) h(x, y) \sin 2 \pi f t]$, where $s(x, y)$ denotes the static object wave front, $h(x, y)$ the vibration amplitude, $\lambda$ the wavelength, and $f$ the vibration frequency. The diffracted field at a distance $d$ from the input plane can be calculated according to Fresnel approximation as:

$$
\begin{aligned}
U(u, v, t) \propto & \exp \left[i \pi \lambda d\left(u^{2}+v^{2}\right)\right] F\{U(x, y, t) \\
& \left.\times \exp \left[i(\pi / \lambda d)\left(x^{2}+y^{2}\right)\right]\right\},
\end{aligned}
$$

where $F$ denotes the Fourier transform operation, $(u, v)$ the spatial frequencies, and where the constant terms are omitted. The exposure is obtained by integrating the instantaneous intensity $|U(u, v, t)|^{2}$, i.e., $E(u, v)=\int_{0}^{\tau}|U(u, v, t)|^{2} \mathrm{~d} t$, where $\tau$ is the exposure time. For the integration time $\tau$ $\gg 1 / f$, one of the additive exposure terms is in the form: ${ }^{8}$

$$
\begin{aligned}
E(u, v) \propto & \exp [i 2 \pi(u X+v Y)] F\left\{s(x, y) J_{0}[(4 \pi / \lambda) h(x, y)]\right. \\
& \left.\times \exp \left[i(\pi / \lambda d)\left(x^{2}+y^{2}\right)\right]\right\},
\end{aligned}
$$

yielding for the reconstruction (modulus of the inverse FT):

$$
\left|U^{\prime}(x, y)\right| \propto|s(x-X, y-Y)|\left|J_{0}[(4 \pi / \lambda) h(x-X, y-Y)]\right| .
$$

In Eqs. (2) and (3), $J_{0}$ is the zero-order Bessel function of the first kind. The Bessel function shows that the object reconstructions are covered with the modulation fringes. The number and distribution of fringes depend on the amplitude $h$, which is generally a function of spatial coordinates and the frequency at which the investigated surface is vibrating, i.e., $h=h(x, y, f)$. Therefore, the hologram reconstruction reveals the main characteristics of the vibration mode: the node structure and vibration amplitudes. In the case of vibrating surfaces of most musical instruments, detection of very regular fringe patterns is expected due to the symmetry of their resonant bodies. For example, irregularities in fringe patterns of percussion instruments indicate detuning of their membranes.

We constructed an experimental device shown schematically in Fig. 1. It is composed of a laser, a variable beam splitter, a beam collimator, an array sensor, and various

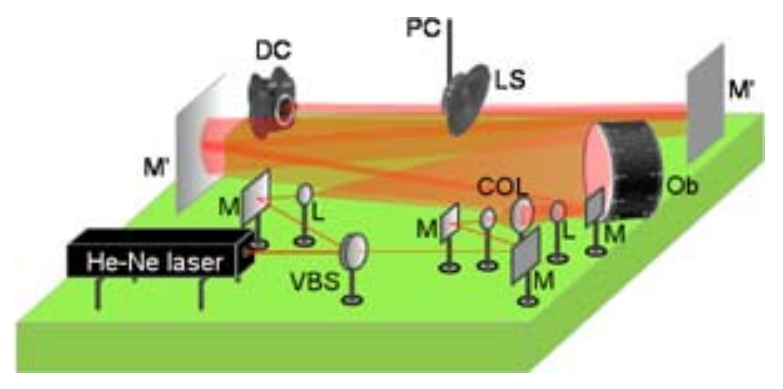

Fig. 1 Scheme of the experimental setup: VBS—variable beam splitter, $\mathrm{M}$-mirror, $\mathrm{M}^{\prime}$-large mirror, $\mathrm{COL}$-collimator, $\mathrm{L}$-lens, VS-vibration surface, LS—loudspeaker, DC—digital camera, PCpersonal computer, and $\mathrm{Ob}$-object. 


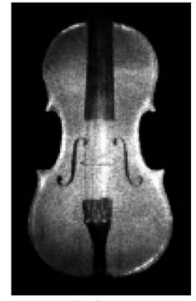

(a)

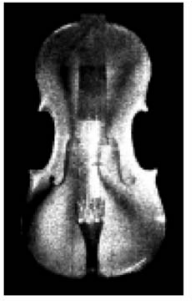

(b)

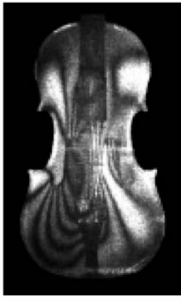

(c)

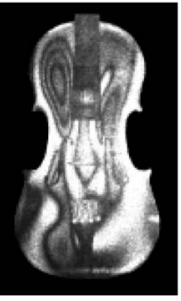

(d)
Fig. 2 Hologram reconstructions for a violin: (a) no excitation, (b) $220 \mathrm{~Hz}$, (c) $523.3 \mathrm{~Hz}$, and (d) $1760 \mathrm{~Hz}$.

lenses and mirrors. We used a He-Ne laser $(\lambda=632.8 \mathrm{~nm})$ as a light source and a color digital camera (Olympus E-1, objective lens removed, sensor: $2560 \times 1920$ pixels of a size $6.8 \times 6.8 \mu \mathrm{m}$, primary RGB filter) as an array photodetector. The beam emerging from the laser is split into an object and a reference beam. The reference beam is first expanded and collimated, then focused in the plane of the object surface, and finally steered by mirrors onto the detector. The object beam is expanded for uniform illumination of the vibrating surface and then directed to the detector. To excite the surface externally, we used a loudspeaker with a sound whose form, frequency, and intensity are controlled by a computer. To shorten the total length of the device, we placed two large inexpensive plane mirrors (size: $400 \times 500 \mathrm{~mm}$ ) between the object and the sensor. Thus, digital holograms are recorded at a distance of $7.5 \mathrm{~m}$ $(3 \times 2.5 \mathrm{~m})$ from the object, using the exposure time of $1 \mathrm{~s}$ and the beam intensity ratio (reference vs. object) between 2.5 and 3.5 .

Each hologram reconstruction is obtained by the following procedure:

(i) the maximum middle square part (1920 $\times 1920$ pixels $)$ of the recorded image (2560 $\times 1920$ pixels) is taken for processing (to preserve the natural proportions of the reconstructed objects),

(ii) the obtained colored image is transformed into an 8-bit gray-scale image,

(iii) inverse Fourier transform operation is performed and its modulus sustained,

(iv) the transparent mask of the object shape is synthesized on the position of the reconstructed object with opaque background,

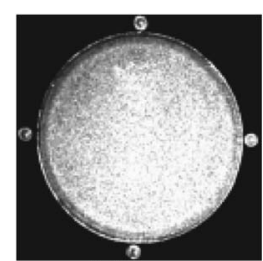

(a)

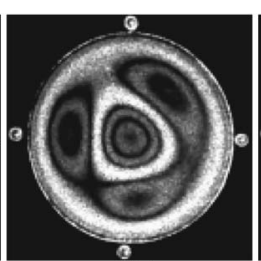

(b)

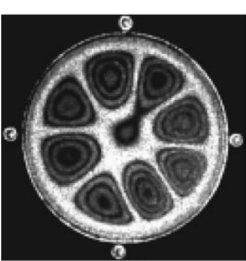

(c)
Fig. 4 Hologram reconstructions for a drum: (a) no excitation, (b) $1040 \mathrm{~Hz}$, and (c) $1415 \mathrm{~Hz}$.

(v) the mask and the reconstructed images are multiplied to select only one reconstruction.

Figures 2-4 demonstrate the experimental results for two types of musical instruments: one type with a string (a violin, Fig. 2) and the other type with a circular membrane (a dumbek, Fig. 3, and a tunable 6-inch drum, Fig. 4). Each figure shows a static hologram reconstruction plus the fringe patterns of typical vibration modes at several frequencies. Figure 5 demonstrates the sensitivity of the method for tuning the drum. The musical instruments with a membrane are tunable insofar as the membrane tension may be adjusted. For an ideal membrane, the accuracy of the tuning is defined by applying identical tension to each lug. The tuning of our drum involves adjusting the tension at four points around its circumference. Figure 5(a) shows symmetrical fringe pattern at the frequency of $440 \mathrm{~Hz}$. By turning only the left lug in steps of quarter turns, we obtained the asymmetrical fringe patterns, as shown in Figs. 5(b)-5(d). By repeating the tuning tests at various frequencies, we observed that the higher frequencies have shown higher tuning sensitivities. That conclusion follows also from the results of the non-tunable instrument. As evident from Fig. 3, while the fringe patterns appear symmetrical at the low frequencies $(220 \mathrm{~Hz}$ and $587.3 \mathrm{~Hz})$, it is apparently asymmetrical at a higher frequency $(1760 \mathrm{~Hz})$.

In conclusion, an inexpensive experimental device for measuring the surface vibrations of musical instruments is reported. The presented results demonstrate the quality of the obtained information about vibration modes and tuning properties of musical instruments. Based on the quasiFourier digital holography setup, the proposed device uses an original architecture compacted with large mirrors and equipped with a commercial color digital camera as an array detector. We have been using two large mirrors, although following the same principle more mirrors can be added to shorten the total length of the device. The notice-

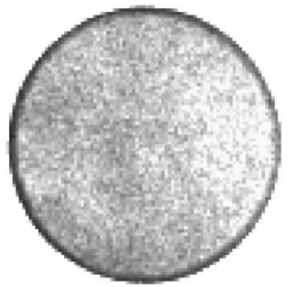

(a)

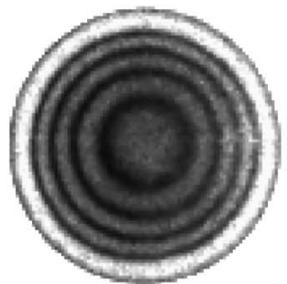

(b)

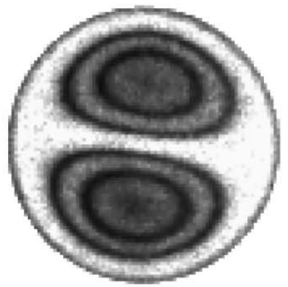

(c)

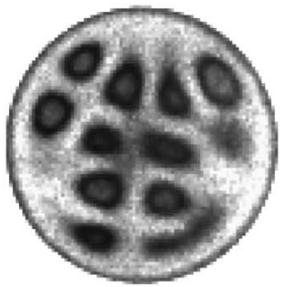

(d)

Fig. 3 Hologram reconstructions for a dumbek: (a) no excitation, (b) $220 \mathrm{~Hz}$, (c) $587.3 \mathrm{~Hz}$, and (d) $1760 \mathrm{~Hz}$ 


\section{OE LETTERS}

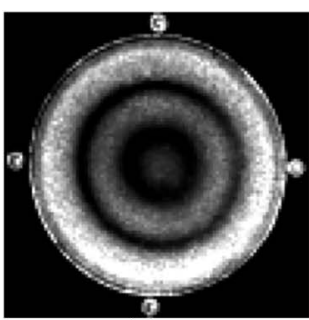

(a)

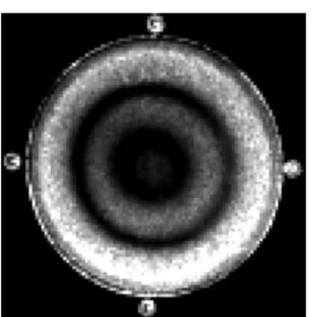

(b)

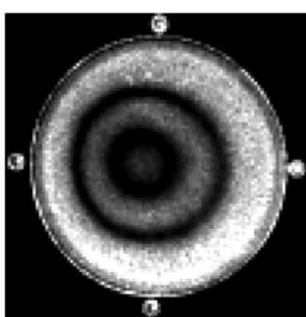

(c)

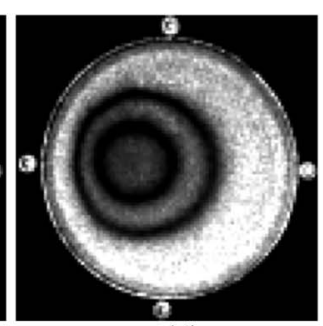

(d)

Fig. 5 Hologram reconstructions for tunning the drum at $440 \mathrm{~Hz}$ : (a) zero state, (b) quarter turn, (c) half turn, and (d)three-quarter turn.

able effect of these inexpensive mirrors was the appearance of the additional fringes of the spatial frequency one order lower than the spatial frequency of the primary interference fringes. However, the quality of the hologram reconstructions was not influenced by these additional fringes. Similarly, the quality of holograms captured by the color digital camera and transformed to gray-scale images was comparable to the quality of holograms captured by a professional monochrome CCD camera regularly used in digital holography. Finally, it is worth noting that an inexpensive commercial device can be constructed for measuring the surface vibrations of a wide class of objects using the principle introduced in this work.

\section{Acknowledgment}

This work was supported by the Croatian Ministry of Science, Education and Sports under the project no. 0035005.

\section{References}

1. N. H. Fletcher and T. D. Rossing, The Physics of Musical Instruments, Springer-Verlag, New York (1998).

2. N. F. Rieger, "The relationship between finite element analysis and modal analysis," Sound Vib. 20, 20-31 (1986).

3. K. D. Marshall, "Modal analysis of a violin," J. Acoust. Soc. Am. 77, 695-709 (1985).

4. W. Reinecke and L. Cremer, "Application of holographic interferometry to vibrations of the bodies of string instruments," J. Opt. Soc. Am. 48, 988-992 (1970).

5. O. J. Lokberg, "ESPI-The ultimate holographic tool for vibration analysis?," J. Acoust. Soc. Am. 75, 1783-1791 (1984).

6. N. Demoli, J. Meštrović, and M. Sović, "Subtraction digital holography," Appl. Opt. 42(5), 798-804 (2003).

7. N. Demoli, D. Vukicevic, and M. Torzynski, "Dynamic digital holographic interferometry with three wavelengths," Opt. Express 11(7), 767-774 (2003).

8. N. Demoli and D. Vukicevic, "Detection of hidden stationary deformations of vibrating surfaces by use of time-averaged digital holographic interferometry," Opt. Lett. 29(20), 2423-2425 (2004). 